University of Texas at El Paso

ScholarWorks@UTEP

$3-2008$

\title{
How to Reconcile Physical Theories with the Idea of Free Will: From Analysis of a Simple Model to Interval and Fuzzy Approaches
}

Julio C. Urenda

Olga Kosheleva

The University of Texas at El Paso, olgak@utep.edu

Follow this and additional works at: https://scholarworks.utep.edu/cs_techrep

Part of the Computer Engineering Commons

Comments:

Technical Report: UTEP-CS-08-15a

Published in Proceedings of the IEEE World Congress on Computational Intelligence WCCI'2008, Hong Kong, China, June 1-6, 2008, pp. 1024-1029.

\section{Recommended Citation}

Urenda, Julio C. and Kosheleva, Olga, "How to Reconcile Physical Theories with the Idea of Free Will: From Analysis of a Simple Model to Interval and Fuzzy Approaches" (2008). Departmental Technical Reports (CS). 82.

https://scholarworks.utep.edu/cs_techrep/82

This Article is brought to you for free and open access by the Computer Science at ScholarWorks@UTEP. It has been accepted for inclusion in Departmental Technical Reports (CS) by an authorized administrator of ScholarWorks@UTEP. For more information, please contact Iweber@utep.edu. 


\title{
How to Reconcile Physical Theories with the Idea of Free Will: From Analysis of a Simple Model to Interval and Fuzzy Approaches
}

\author{
Julio C. Urenda and Olga Kosheleva
}

\begin{abstract}
Most modern physical theories are formulated in terms of differential equations. As a result, if we know exactly the current state of the world, then this state uniquely determines all the future events - including our own future behavior. This determination seems to contradict the intuitive notion of a free will, according to which we are free to make decisions - decisions which cannot be determined based on the past locations and velocities of the elementary particles. In quantum physics, the situation is somewhat better in the sense that we cannot determine the exact behavior, but we can still determine the quantum state, and thus, we can determine the probabilities of different behaviors - which is still inconsistent with our intuition.

This inconsistency does not mean, of course, that we can practically predict our future behavior; however, in view of many physicists and philosophers, even the theoretical inconsistency is somewhat troubling. Some of these researchers feel that it is desirable to modify physical equations in such a way that such a counter-intuitive determination would no longer be possible.

In this paper, we analyze the foundations for such possible theories, and show that on the level of simple mechanics, the formalization of a free will requires triple interactions - while traditional physics is based on pairwise interactions between the particles.
\end{abstract}

\section{FREE WILL: A NATURAL IDEA}

Intuitively, most of us believe that we are able to make conscientious decisions, i.e., that we have free will. If we walk to a corner, then we can turn right or cross the street. The commonsense belief is that it is not possible to predict beforehand what exactly a person will do.

\section{THERE SEEMS TO BE NO FREE WILL IN CLASSICAL} MECHANICS (AND IN CLASSICAL PHYSICS IN GENERAL)

In contrast to the common sense belief, in classical physics, e.g., in classical mechanics where Newton's equations describe the motion of all the particles, once we know the positions and velocities of all the particles, we can uniquely predict the exact future locations and velocities of all the particles.

Julio C. Urenda is with the Department of Mathematics, and Olga Kosheleva is with the Department of Teacher Education, University of Texas at El Paso, emails jcurenda@miners.utep.edu, olgak@utep.edu.

This work was supported in part by NSF grants HRD-0734825, EAR0225670, and EIA-0080940, by Texas Department of Transportation grant No. 0-5453, by the Japan Advanced Institute of Science and Technology (JAIST) International Joint Research Grant 2006-08, and by the Max Planck Institut für Mathematik. The authors are grateful to Professor John Symons from Philosophy Department for valuable discussions.

The authors are thankful to the anonymous referees for valuable discussions.

\author{
III. CLARIFICATION: PREDICTIONS ARE NOT \\ PRACTICALLY POSSIBLE, BUT EVEN THE THEORETICAL \\ POSSIBILITY OF PREDICTION IS VERY \\ COUNTER-INTUITIVE
}

Of course, with $\approx 10^{23}$ particles in a human being (see, e.g., [10]), it is not practically possible to actually solve the corresponding system of equations, so this possibility of prediction remains a purely theoretical possibility. However, from the commonsense viewpoint, even a theoretical prediction probability is very disturbing: it says, in effect, that we do not have any free will, all our words and movements are uniquely determined by the original state of the Universe several billion years ago.

\section{CAN WE RECONCILE PHYSICS WITH FREE WILL?}

The possibility to predict every single detail of our behavior (even theoretically) is so contrary to our intuition that it is natural to ask: is it possible to modify physical equations in such a way that there will be room for free will?

\section{IS QUANTUM PHYSICS AN ANSWER?}

At first glance, it may look as if this problem disappears in quantum physics. Indeed, in quantum mechanics, due to Heisenberg's principle, we cannot exactly predict both the location and the velocity of a particle, we can only predict the probabilities of different values of location and velocity.

However, even in quantum mechanics, Schroedinger's equations which describe how the state ("wave function") $\psi(x, t)$ changes with time $t$ are deterministic. Once we know the original state $\psi\left(x, t_{0}\right)$ at some moment $t_{0}$ in the past, we can uniquely determine the state at all future moments $t>t_{0}$. Yes, knowing the state $\psi(x, t)$ does not allow us to uniquely predict the observed values, but it does enables us to uniquely predict the probabilities of different observed values.

In terms of human behavior, this means that we cannot predict whether a person turns or not, but we can predict (at least theoretically) the probability that a person turns right. This also does not seem to be consistent with common sense.

\section{THE PROBLEM OF FREE WILL IN PHYSICS HAS BEEN ACTIVELY STUDIED IN PHILOSOPHY}

The seeming disconnect between physics and the intuitive notion of a free will has been actively studied in philosophy, especially by philosophers and physicists interested in foundations of physics; see, e.g., [5], [6], [7], [8], [11], [12], [15], [16], [29], [30], [32], [33], [35], [36] and references therein. 
There seem to be two main ways of resolving this seeming contradiction between physics and common sense intuition:

- The mainstream approach, advocated by the majority of physicists, is to keep the physics as is. In this approach, our commonsense intuition is considered to be faulty - just like quantum mechanics showed that a lot of other commonsense intuitions are only approximately correct; see, e.g., [10]. A good argument in favor of this approach is that

- while theoretically, we can predict a human behavior,

- in practice, this would require a practically impossible accuracy in knowing the initial conditions of all the particles, and a practically impossible amount of information (which is needed to store all the information about these initial states).

- However, several philosophically minded physicists, including Penrose [29], feel that they will be more comfortable with a physical theory which is consistent with our intuition about the free will, consistent both on the practical level (as existing theories are) and on the theoretical philosophical level.

\section{AT PRESENT, THERE IS NO CONVINCING WELL-DEVELOPED PHYSICAL THEORY WHICH IS FULLY CONSISTENT WITH OUR FREE WILL INTUITION}

At present, most physical theories are described in terms of differential equations - just like they have been described since Newton's breakthrough equations of motion. Differential equations usually have the property that once we know their initial state, we can then uniquely determine the state at all the following moments of time - thus violating our intuition about the free will.

From the purely mathematical viewpoint, there are techniques which allow non-unique predictions, such as differential inclusions (see, e.g., [2], [31]), where we only predict some properties of the future state.

\section{INTERVAL AND FUZZY APPROACHES: TOWARDS} RECONCILIATION BETWEEN PHYSICS AND FREE WILL

Specifically, in the standard differential equations, once we know the initial state, we can uniquely predict the rate with which this state changes - and thus, uniquely predict state at all future moments of time.

For example, in classical mechanics, the state is determined by the locations and velocities of all the particles. In such theories, the rate with which the state change is the acceleration of the particles. Indeed, Newton's mechanics uniquely determines this acceleration $\vec{a}_{i} \stackrel{\text { def }}{=} \frac{d \vec{v}_{i}}{d t}$ of the $i$ th particle as a function of the current coordinates $\vec{r}_{j}$ and velocities $\vec{v}_{j}$ of all the particles:

$$
\frac{d \vec{v}_{i}}{d t}=\vec{F}_{i}\left(\vec{r}_{1}, \ldots, \vec{r}_{n}, \vec{v}_{1}, \ldots, \vec{v}_{n}\right),
$$

for some (known) function $\vec{F}_{i}\left(\vec{r}_{1}, \ldots, \vec{r}_{n}, \vec{v}_{1}, \ldots, \vec{v}_{n}\right)$. This means that each coordinate $a_{i a}$ of the acceleration vector $\vec{a}_{i}$ is determined by the formula

$$
\frac{d v_{i a}}{d t}=F_{i a}\left(\vec{r}_{1}, \ldots, \vec{r}_{n}, \vec{v}_{1}, \ldots, \vec{v}_{n}\right) .
$$

In the alternative approaches, even when we know the initial state, we do not know the exact value of the statechanging rate. For each component of this rate, we may only know the interval of possible values. In other words, instead of the above differential equation, we have have a differential inclusion (see, e.g., [2], [31]

$$
\frac{d v_{i a}}{d t} \in\left[\underline{F}_{i a}\left(\vec{r}_{1}, \ldots, \vec{r}_{n}, \vec{v}_{1}, \ldots\right), \bar{F}_{i a}\left(\vec{r}_{1}, \ldots, \vec{r}_{n}, \vec{v}_{1}, \ldots\right)\right]
$$

for some (known) functions $\underline{F}_{i a}\left(\vec{r}_{1}, \ldots, \vec{r}_{n}, \vec{v}_{1}, \ldots, \vec{v}_{n}\right)$ and $\bar{F}_{i a}\left(\vec{r}_{1}, \ldots, \vec{r}_{n}, \vec{v}_{1}, \ldots, \vec{v}_{n}\right)$. In this case, the actual value of the acceleration is now known, it can take any value from the (known) interval

$$
\left[\underline{F}_{i a}\left(\vec{r}_{1}, \ldots, \vec{r}_{n}, \vec{v}_{1}, \ldots, \vec{v}_{n}\right), \bar{F}_{i a}\left(\vec{r}_{1}, \ldots, \vec{r}_{n}, \vec{v}_{1}, \ldots, \vec{v}_{n}\right)\right] .
$$

It is also possible that instead of a single interval describing possible values of the acceleration, we have several intervals corresponding to different degrees of certainty. Such nested intervals can be viewed as $\alpha$-cuts of a fuzzy set; see, e.g., [9], [18], [24], [27], [28].

For details on the resulting fuzzy differential inclusions, see, e.g., [20].

\section{LIMITATIONS OF THE EXISTING APPROACHES}

Techniques based on differential inclusions, intervals, and fuzzy uncertainty are much less developed (both mathematically and computationally) than differential equations and thus, much rarer used in physics.

\section{What WE PLAN TO DO}

The main objective of this paper is to contribute to the analysis of possible free-will-allowing physical theories. As we have mentioned, such analysis has been done before, usually via discussions on how complex quantum theories can be appropriately modified.

In this paper, we plan to approach this problem from a different prospective: from the prospective of foundations of physics. From this viewpoint, instead of starting with complex modern physical theories, we start with basic physical equations.

Specifically, we start with the simplest mechanics-type physical theories, where we have a small number of particles moving and interacting with each other - in the style of Newtonian mechanics.

Moreover, we will start with the simplest subcase of this simplest case, when we have point-size bodies which do not interact with each other at all.

In principle, bodies which do not have any Newtonstyle interaction-at-a-distance can still bump into each other, However, since we assumed that these 3-D bodies are pointsize, they need to be moving exactly toward the same point for them to hit each other. Even a small deviation would make them miss each other. Thus, if we assume that the particles are in generic positions, we can safely assume that these bodies do not bump into each other at all. 


\section{TRADITIONAL DESCRIPTION OF THIS SITUATION}

In the traditional description of this situation, we have several bodies which do not interact with each other. Because of this non-interaction, each body follows a straight-line trajectory which is uniquely determine by its initial location and initial velocity.

\section{What We Plan to DESCRIBE}

Our objective is to see how can we reasonably modify the equations of physics so that it will be possible to make their motion no longer uniquely predictable.

In plain terms, a physically explicit free will would mean that by simply exercising our will, we can actually change the motion of the physical particles. We would like to check if this is indeed possible within a meaningful physical theory.

\section{OUR MAIN ASSUMPTION: THE PHYSICAL THEORY MUST BE CONSISTENT WITH NATURAL PHYSICAL THEORIES AND THUS, CONSISTENT WITH CONSERVATION LAWS}

Of course, we could have all kinds of mathematically reasonable "physical theories" in which particles move in whatever direction we want them do. What we want is a theory which is physically meaningful.

What makes a theory physically meaningful? In modern physics, one of the most important notions is the notion of symmetry; see, e.g., [10]. The behavior of the physical particles must not change if we simply shift them to a different spatial location, or rotate the whole configuration, or start the experiment at a later time moment. In precise terms, the equations of physics must be invariant with respect to main geometric symmetries such as shift in space, shift in time, and rotation.

It is known that in physical equations, invariance with respect to symmetries lead to conservation laws [10]:

- invariance with respect to shifts in time means that energy must be preserved;

- invariance with respect to spatial shifts means that the (linear) momentum must be preserved, and

- invariance with respect to rotations means that the angular momentum must be preserved.

Thus, we require that these three quantities are preserved in our physical theory. Let us formulate this requirement in precise terms.

\section{INVARIANCE IN PRECISE TERMS}

Let $m_{i}$ denote the mass of the $i$-th particle, $\vec{r}_{i}$ denote its location at a given moment of time, and let $\vec{v}_{i}$ denote this particle's velocity. Energy conservation means that the overall energy

$$
E=\sum_{i=1}^{n} \frac{1}{2} \cdot m_{i} \cdot\left(\vec{v}_{i}\right)^{2}
$$

must be preserved, i.e., that its value must be the same at all moments of time. Momentum conservation means that the overall (linear) momentum

$$
\vec{p}=\sum_{i=1}^{n} m_{i} \cdot \vec{v}_{i}
$$

must be preserved, and the conservation of the angular momentum means that the overall angular momentum

$$
\vec{M}=\sum_{i=1}^{n} m_{i} \cdot\left(\vec{v}_{i} \times \vec{r}_{i}\right),
$$

where $\vec{a} \times \vec{b}$ denotes the vector product of the two vectors. As usual, we also assume that the masses $m_{i}$ of the particles do not change with time.

\section{CASE OF A Single PaRTicle}

Let us start our analysis with the case of a single particle. For this particle, from the fact that the momentum $\vec{p}=m_{1} \cdot \vec{v}_{1}$ is preserved and that the mass $m_{1}$ is preserved, we conclude that the velocity $\vec{v}_{1}$ should also be preserved. In other words, if we have a single particle which moves with a velocity $\vec{v}_{1}$, it will continue moving with this same velocity.

So, for a single particle, no "true free-will" theory is possible: no matter how much we exercise our will, this particle will not be diverted from its inertial path.

\section{CASE OF TWO PARTICLES: ANALYSIS}

The next case is the case when we have two particles. To make the analysis easier (and more transparent), let us select a coordinate system appropriately. Specifically, as the origin $\overrightarrow{0}$ of the coordinate system, we select the center of gravity $\frac{m_{1} \cdot \vec{r}_{1}+m_{2} \cdot \vec{r}_{2}}{m_{1}+m_{2}}$ of the 2-particle system. In other words, the two particles must satisfy the condition

$$
m_{1} \cdot \vec{r}_{1}+m_{2} \cdot \vec{r}_{2}=\overrightarrow{0} .
$$

The speed $\vec{v}$ with which the location of the center of gravity changes in time can be described as

$$
\vec{v}=\frac{d}{d t}\left(\frac{m_{1} \cdot \vec{r}_{1}+m_{2} \cdot \vec{r}_{2}}{m_{1}+m_{2}}\right)=\frac{m_{1} \cdot \vec{v}_{1}+m_{2} \cdot \vec{v}_{2}}{m_{1}+m_{2}},
$$

i.e., as $\vec{v}=\frac{\vec{p}}{m_{1}+m_{2}}$. Since the momentum

$$
\vec{p}=m_{1} \cdot \vec{v}_{1}+m_{2} \cdot \vec{v}_{2}
$$

is preserved, and the masses $m_{1}$ and $m_{2}$ also do not change with time, the speed $\vec{v}$ is also preserved. We can therefore select a new coordinate system which moves with this constant velocity $\vec{v}$ with respect to the original one. In this new coordinate system, we have $\vec{v}=0$, i.e.,

$$
m_{1} \cdot \vec{v}_{1}+m_{2} \cdot \vec{v}_{2}=0 .
$$

From this, we conclude that

$$
\vec{v}_{2}=-\frac{m_{1}}{m_{2}} \cdot \vec{v}_{1} .
$$

Now, we are ready for the desired analysis. In the traditional physics, both non-interacting particle preserve their inertial motion, i.e., both velocities $\vec{v}_{1}$ and $\vec{v}_{2}$ do not change 
with time, and their accelerations $\vec{a}_{i} \stackrel{\text { def }}{=} \frac{d \vec{v}_{i}}{d t}$ are equal to 0. We are interested in the situations in which at least one of the two particles deviates from its original inertial trajectory, i.e., at least one of the accelerations $\vec{a}_{i}$ is different from 0 .

We assumed that the momentum is preserved, i.e., that $\vec{p}=m_{1} \cdot \vec{v}_{1}+m_{2} \cdot \vec{v}_{2}$ does not change with time: $\frac{d \vec{p}}{d t}=0$. Differentiating the expression $\vec{p} \vec{p}=m_{1} \cdot \vec{v}_{1}+m_{2} \cdot \vec{v}_{2}$ with respect to time, we conclude that $\frac{d \vec{p}}{d t}=m_{1} \cdot \vec{a}_{1}+m_{2} \cdot \vec{a}_{2}=0$. Thus,

$$
\vec{a}_{2}=-\frac{m_{1}}{m_{2}} \cdot \vec{a}_{1} .
$$

Similarly, from the fact that the angular momentum is preserved, we conclude that

$$
m_{1} \cdot\left(\vec{a}_{1} \times \vec{r}_{1}\right)+m_{2} \cdot\left(\vec{a}_{2} \times \vec{r}_{2}\right)=0 .
$$

We know that $\vec{a}_{2}=-\frac{m_{1}}{m_{2}} \cdot \vec{a}_{1}$, and from the fact that we have a coordinate system with the origin at the center of gravity, we conclude that $\vec{r}_{2}=-\frac{m_{1}}{m_{2}} \cdot \vec{r}_{1}$. Substituting these expressions for $\vec{a}_{2}$ and $\vec{r}_{2}$ into the above formula, we conclude that

$$
m_{1} \cdot\left(\vec{a}_{1} \times \vec{r}_{1}\right)+m_{2} \cdot\left(\frac{m_{1}}{m_{2}}\right)^{2} \cdot\left(\vec{a}_{1} \times \vec{r}_{1}\right)=0,
$$

i.e., that

$$
\left(m_{1}+\frac{m_{1}^{2}}{m_{2}}\right) \cdot\left(\vec{a}_{1} \times \vec{r}_{1}\right)=0
$$

and hence that $\vec{a}_{1} \times \vec{r}_{1}=0$. The vector product is equal to 0 only when the vectors are collinear, i.e., when $\vec{a}_{1} \| \vec{r}_{1}$. In other words, $\vec{a}_{1}=\alpha \cdot \vec{r}_{1}$ for some real number $\alpha$. Since we assumed that at least one of the particles has a non- 0 acceleration, we must have $\alpha \neq 0$.

Since the energy

$$
E=\frac{1}{2} \cdot m_{1} \cdot\left(\vec{v}_{1}\right)^{2}+\frac{1}{2} \cdot m_{2} \cdot\left(\vec{v}_{2}\right)^{2}
$$

is also preserved, its derivative with respect to time is also equal to 0 :

$$
m_{1} \cdot\left(\vec{v}_{1} \cdot \vec{a}_{1}\right)+m_{2} \cdot\left(\vec{v}_{2} \cdot \vec{a}_{2}\right)=0,
$$

where $\vec{a} \cdot \vec{b}$ denotes a scalar (dot) product of the two vectors. We know that $\vec{v}_{2}=-\frac{m_{1}}{m_{2}} \cdot \vec{v}_{1}$ and $\vec{a}_{2}=-\frac{m_{1}}{m_{2}} \cdot \vec{a}_{1}$, hence we get $\vec{a}_{1} \cdot \vec{v}_{1}=0$. Since $\vec{a}_{1}=\alpha \cdot \vec{r}_{1}$, we conclude that $\vec{r}_{1} \cdot \vec{v}_{1}=0$.

We started this analysis by assuming that the particles are in a generic position. In general, the scalar product $\vec{r}_{1} \cdot \vec{v}_{1}$ can attain any real value and thus, in the generic case, it cannot be equal to 0 .

So, we arrive at the following conclusion.

\section{CASE OF TWO PARTICLES: CONCLUSION}

For two particles, from the fact that the momentum, energy, and angular momentum are preserved, we conclude that in the generic case, both velocities $\vec{v}_{1}$ and $\vec{v}_{2}$ are also preserved. In other words, if we have two particles in a general position which move with velocities $\vec{v}_{1}$ and $\vec{v}_{2}$, they will continue moving with the same velocities.

So, for two particles, no "true free-will" theory is possible: no matter how much we exercise our will, these particles will not be diverted from their inertial paths.

Comment. Instead of selecting a coordinate system in which computations are easier, we could as well perform all the computations in the original (arbitrary) coordinate system and attain the same conclusions.

\section{CASE OF THREE (OR MORE) PARTICLES: ANALYSIS}

For the case of three particles, the need to preserve energy, momentum, and angular momentum leads to the following equations for the three unknown accelerations $\vec{a}_{1}, \vec{a}_{2}$, and $\vec{a}_{3}$ :

$$
\begin{gathered}
m_{1} \cdot \vec{a}_{1}+m_{2} \cdot \vec{a}_{2}+m_{3} \cdot \vec{a}_{3}=0 \\
m_{1} \cdot\left(\vec{a}_{1} \times \vec{r}_{1}\right)+m_{2} \cdot\left(\vec{a}_{2} \times \vec{r}_{2}\right)+m_{3} \cdot\left(\vec{a}_{3} \times \vec{r}_{3}\right)=0 ; \\
m_{1} \cdot\left(\vec{a}_{1} \cdot \vec{v}_{1}\right)+m_{2} \cdot\left(\vec{a}_{2} \cdot \vec{v}_{2}\right)+m_{3} \cdot\left(\vec{a}_{3} \cdot \vec{v}_{3}\right)=0 .
\end{gathered}
$$

Let us analyze this system of equations. We need to find three 3 -D vectors $\vec{a}_{1}, \vec{a}_{2}$, and $\vec{a}_{3}$, i.e., $3 \cdot 3=9$ scalar unknowns.

How many scalar equations do we have? The first two equations are vector equations, each of which has 3 scalar components; so overall, we have 7 scalar equations to determine 9 (scalar) unknowns. Clearly, a linear system of 7 equations with 9 unknowns has many solutions.

For $p>3$ particles, we need $3 \cdot p$ scalar parameters to determine $p$ accelerations $\vec{a}_{1}, \ldots, \vec{a}_{p}-$ and the same number of 7 equations to satisfy. Since $3 \cdot p>3 \cdot 3>7$, the corresponding linear system of equations always has a nonzero solution $\vec{a}_{i}$.

\section{CASE OF THREE (OR MORE) PARTICLES: CONCLUSION}

For three (or more) particles, a "true free-will" physical theory is, in principle, possible.

Comment. For two particles, we need to determine 6 parameters - and we have $7>6$ equations that they must satisfy. In general, an over-determined system of 7 linear equations with 6 unknowns does not have a non-zero solution. No wonder that for $p=2$, we could not find such a solution.

\section{General CONCLUSION}

Most physical theories such as gravity or electrodynamics are based on pairwise interactions between particles. For example, in Newton's gravitation theory, a force $\vec{F}_{i}=m_{i} \cdot \vec{a}_{i}$ acting on the $i$-th body is equal to the sum of the corresponding pairwise forces:

$$
\vec{F}_{i}=\sum_{j \neq i} \frac{G \cdot m_{i} \cdot m_{j} \cdot\left(\vec{r}_{j}-\vec{r}_{i}\right)}{\left|\vec{r}_{j}-\vec{r}_{i}\right|^{3}} .
$$

In such theories, there is also a complex interaction between three or more bodies. For example, tides are a good example of a gravitational interaction between the Earth, the 
Sun, and the Moon. However, on the fundamental level, all this complex interaction can be reduced to (and explained by) pairwise interactions.

Our simple model seems to indicate that if we only consider pairwise interactions, we will never come up with a true free-will theory. To come up with such a theory, we must consider a fundamentally different physical theory, in which there are triple (or higher order) interactions between particles which cannot be reduced to their pairwise interactions.

In other words, instead of the usual formula

$$
\vec{F}_{i}=\sum_{j \neq i} \vec{F}_{i j}\left(\vec{r}_{i}, \vec{r}_{j}, \vec{v}_{i}, \vec{v}_{j}\right)
$$

which only takes into account pair-wise interactions, we should try to get a more general formula

$$
\begin{gathered}
\vec{F}_{i}=\sum_{j \neq i} \vec{F}_{i j}\left(\vec{r}_{i}, \vec{r}_{j}, \vec{v}_{i}, \vec{v}_{j}\right)+ \\
\sum_{j \neq i} \sum_{k \neq i} \vec{F}_{i j k}\left(\vec{r}_{i}, \vec{r}_{j}, \vec{r}_{k}, \vec{v}_{i}, \vec{v}_{j}, \vec{v}_{k}\right)
\end{gathered}
$$

that also takes into account ternary interactions - or maybe even interactions of higher order:

$$
\begin{gathered}
\vec{F}_{i}=\sum_{j \neq i} \vec{F}_{i j}\left(\vec{r}_{i}, \vec{r}_{j}, \vec{v}_{i}, \vec{v}_{j}\right)+ \\
\sum_{j \neq i} \sum_{k \neq i} \vec{F}_{i j k}\left(\vec{r}_{i}, \vec{r}_{j}, \vec{r}_{k}, \vec{v}_{i}, \vec{v}_{j}, \vec{v}_{k}\right)+ \\
\sum_{j \neq i} \sum_{k \neq i} \sum_{l \neq i} \vec{F}_{i j k}\left(\vec{r}_{i}, \vec{r}_{j}, \vec{r}_{k}, \vec{r}_{l}, \vec{v}_{i}, \vec{v}_{j}, \vec{v}_{k}, \vec{v}_{l}\right)+
\end{gathered}
$$

\section{DIscussion}

In short, to formulate a non-trivial free-will theory, we need at least triple interactions. This conclusion is in good accordance with other situations where a problem is easy under pairwise interactions but becomes much less trivial under triple ones:

- in group decision making, it is easy to combine the opinions of two people, but combining the opinions of three or more participants leads to known problems related to Arrow's paradox; see, e.g., [1], [17], [21], [25], [26];

- in celestial mechanics, 2-body problem can be explicitly solved while a 3-body problem is much more computationally complicated; see, e.g., [3], [14].

This conclusion has an interesting relation to fuzzy logic. In the traditional fuzzy logic, we have only binary logical operations - the fuzzy analogues of standard logical operations like "or", "and", etc. More complex logical operations (e.g., ternary one) are usually reduced to the binary ones. In [4], [13], [19], [22], [23], [34], it is argued that if we represent such ternary operations directly, then we may get a more adequate representation of expert uncertainty.

It would be nice to find a way use these operations on exact original states, and then predict future states under uncertainty.

\section{REFERENCES}

[1] K. J. Arrow, Social Choice and Individual Values, Wiley, New York, 1951.

[2] J.-P. Aubin and A. Cellina, Differential Inclusions: Set-Valued Maps and Viability Theory, Springer Verlag, Berlin-Heidelberg, 1984.

[3] D. Boccaletti and G. Pucacco, Theory of Orbits, Springer-Verlag, Berlin, Heidelberg, New York, 1998.

[4] B. Bouchon-Meunier, V. Kreinovich, and H. T. Nguyen, "NonAssociative Operations", Proceedings of the Second International Conference on Intelligent Technologies InTech'2001, Bangkok, Thailand, November 27-29, 2001, pp. 39-46.

[5] F. Crick, The Astonishing Hypothesis: The Scientific Search for the Soul, Charles Scribner \& Sons, New York, 1993.

[6] P. Davies, God and the New Physics, Simon \& Schuster, New York, 1983.

[7] D. Dennet, Elbow Room, MIT Press, Cambridge, Massachusetts, 1984.

[8] D. Dennett, Freedom Evolves, Viking Penguin, New York, 2003.

[9] D. Dubois and H. Prade, Operations on fuzzy numbers, International Journal of Systems Science, 1978, Vol. 9, pp. 613-626.

[10] R. Feynman, R. Leighton, and M. Sands, Feynman Lectures on Physics, Addison Wesley, 1970.

[11] J. M. Fischer, God, Foreknowledge and Freedom, Stanford University Press, Stanford, California, 1989.

[12] H. Frankfurt, "Alternate possibilities and moral responsibility", Journal of Philosophy, vol. 66, pp. 829-839, 1969.

[13] I. R. Goodman, R. A. Trejo, V. Kreinovich, J. Martinez, and R. Gonzalez, "An even more realistic (non-associative) interval logic and its relation to psychology of human reasoning", Proceedings of the Joint 9th World Congress of the International Fuzzy Systems Association and 20th International Conference of the North American Fuzzy Information Processing Society IFSA/NAFIPS 2001, Vancouver, Canada, July 25-28, 2001, pp. 1586-1591.

[14] Y. Hagihara, Celestial Mechanics, MIT Press, Cambridge, Massachusetts, 1970.

[15] J. Hospers, "What Means this Freedom?", In: S. Hook (ed.), Determinism and Freedom in the age of modern science, Collier-Macmillan, New York, 1958, pp. 126-142.

[16] R. Kane, The Oxford Handbook of Free Will, Oxford University Press, New York, 2002.

[17] P. R. Kleindorfer, H. C. Kunreuther, and P. J. H. Schoemaker, Decision Sciences: An Integrated Perspective, Cambridge Universoty Press, Cambridge, Massachusetts, 1993.

[18] G. Klir and B. Yuan, Fuzzy sets and fuzzy logic, Prentice Hall, New Jersey, 1995.

[19] V. Kreinovich, "Towards More Realistic (e.g., Non-Associative) 'and'and 'or'-Operations in Fuzzy Logic", Soft Computing, vol. 8, no. 4, pp. 274-280, 2004

[20] V. Lakshmikantham and R. N. Mohapatra, Theory of Fuzzy Differential Equations and Inclusions, CRC Press, Boca Raton, Florida, 2003.

[21] D. R. Luce and H. Raiffa, Games and Decisions, Introduction and critical survey, John Wiley \& Sons, Inc., New York, 1957.

[22] J. Martinez, Towards more realistic (e.g. non-associative) "and"and "or"-operations in fuzzy logic, Master's Thesis, Department of Computer Science, University of Texas at El Paso, 2002.

[23] J. Martinez, L. Macias, A. Esper, J. Chaparro, V. Alvarado, S. A. Starks, and V. Kreinovich, "Towards more realistic (e.g., nonassociative) and- and or-operations in fuzzy logic", Proceedings of the 2001 IEEE Systems, Man, and Cybernetics Conference, Tucson, Arizona, October 7-10, 2001, pp. 2187-2192.

[24] R. E. Moore and W. Lodwick, Interval Analysis and Fuzzy Set Theory, Fuzzy Sets and Systems, 2003, Vol. 135, No. 1, pp. 5-9.

[25] R. B. Myerson, Game theory. Analysis of conflict, Harvard University Press, Cambridge, MA, 1991.

[26] H. T. Nguyen, O. Kosheleva, and V. Kreinovich, "Decision Making Beyond Arrow's 'Impossibility Theorem', With the Analysis of Effects of Collusion and Mutual Attraction", Proceedings of the Sixth International Conference on Intelligent Technologies InTech'05, Phuket Island, Thailand, December 14-16, 2005, pp. 43-52.

[27] H. T. Nguyen and V. Kreinovich, Nested Intervals and Sets: Concepts, Relations to Fuzzy Sets, and Applications, In: R. B. Kearfott and V. Kreinovich, eds., Applications of Interval Computations, Kluwer, Dordrecht, 1996, pp. 245-290.

[28] H. T. Nguyen and E. A. Walker, A First Course in Fuzzy Logic, CRC Press, Boca Raton, Florida, 2006. 
[29] R. Penrose, A. Shimony, N. Cartwright, S. Hawking, and M. Longair, The Large, the Small and the Human Mind, Cambridge University Press, Cambridge, Massachusetts, 2000.

[30] S. Smilansky, Free Will and Illusion, Oxford University Press, New York, 2000.

[31] G. V. Smirnov, Introduction to the Theory of Differential Inclusions, American Mathematical Society, Providence, Rhode Island, 2002.

[32] P. Suppes, "The Transcendental Character of Determinism," Midwest Studies in Philosophy, vol. 18, pp. 242-257, 1993.

[33] F. J. Tipler, The Physics of Immortality: Modern Cosmology, God and the Resurrection of the Dead, Doubleday, New York, 1994.
[34] R. Trejo, V. Kreinovich, I. R. Goodman, J. Martinez, and R. Gonzalez, "A Realistic (Non-Associative) Logic And a Possible Explanations of $7 \pm 2$ Law", International Journal of Approximate Reasoning, vol. 29, pp. 235-266, 2002

[35] J. Trusted, Free Will and Responsibility, Oxford University Press, New York, 1984.

[36] P. Van Inwagen, An Essay on Free Will, Clarendon Press, Oxford, 1983. 